\title{
Edito x ellas: una app que conecta educación e igualdad de género
}

\author{
Edit for her: an app that connects education \\ and gender equality
}

\section{Maday Domínguez Sánchez \\ Universidad de Las Palmas de Gran Canaria, España \\ E-mail: maday26213@ @otmail.com}

\author{
Arminda Álamo Bolaños \\ Profesora Contratada Doctora \\ Dpto. de Educación. Área de Teoría e Historia de la Educación \\ Universidad de Las Palmas de Gran Canaria, España \\ E-mail: arminda.alamo@ulpgc.es
}

\begin{abstract}
Resumen: Las distintas esferas de la sociedad contemporánea continúan atravesadas por situaciones que responden a una estructura estrictamente patriarcal. En este sentido, los centros escolares tienen un papel fundamental en la transmisión de normas, creencias y valores que contribuyen al constructo identitario del alumnado. Sin embargo, las acciones pedagógicas que se desarrollan en algunas escuelas tienden a reproducir, de manera implícita, elementos atravesados por la desigualdad entre los géneros. El objetivo de esta investigación fue indagar sobre los aspectos que se transmiten en el aula a través del estudio del currículo oculto de los libros de texto en Educación Primaria. La metodología implementada implicó la investigación y desarrollo, un análisis mixto cuantitativo y cualitativo, y el diseño de una aplicación para dispositivos móviles, "Edito X Ellas", que
\end{abstract}


permitió el reporte del contenido sexista presente en los libros de texto. Los resultados evidenciaron la existencia de diversas prácticas que nos indicaron que existía la necesidad de trabajar conjuntamente con las editoriales, los contenidos de sus libros a través de la utilización de "Edito X Ellas". Las conclusiones nos mostraron la importancia del papel del profesorado para la detección de estos aspectos machistas y la promoción de la igualdad desde el currículum de Educación Primaria.

Palabras clave: Aplicación, Igualdad, Curriculum oculto, Libros de Texto.

\begin{abstract}
The different spheres of contemporary society continue to be traversed by situations that respond to a strictly patriarchal structure. In this sense, schools have a fundamental role in the transmission of the norms, beliefs and values that have given rise to the identity construct of the students. However, the pedagogical actions that have been seen in some schools to reproduce, in an implicit way, elements traversed by a patriarchal culture. The objective of this research was on the aspects that are transmitted in the classroom through the study of the textbooks in Primary Education. The methodology implemented involved research and development, a mixed quantitative and qualitative analysis, and the design of an application for mobile devices, Edit For Her, the report of the sexist content present in textbooks. The results showed the existence of various practices that indicate that there is a need to work with publishers, the contents of their books through the use of Edit For Her.
\end{abstract}

The conclusions showed us the importance of the role of teachers in the detection of these «machista» aspects and the promotion of equality from the curriculum of Primary Education.

Keywords: Application, Equality, Hidden Curriculum, Textbooks. 


\section{Introducción}

En una sociedad plural, en constante cambio, se hace imprescindible promover una educación para la igualdad de géneros, debiendo prestar especial atención a aquellas ideas que se transmiten de manera implícita e inconsciente. Sin embargo, las acciones pedagógicas que se llevan a cabo en los centros educativos tienden a reproducir prácticas educativas atravesadas por una cultura patriarcal.

Casares (2008), afirma, en esta línea, que el hombre sigue dominando y oprimiendo a la mujer con diversas formas de violencia, siendo visibles en los distintos ámbitos de la escuela (elección de compañeros para jugar un partido, creencia de los niños de debilidad si están rodeados de muchas niñas...).

Así mismo, el sistema económico retroalimenta al patriarcado al atribuirle a la mujer un papel secundario como consumidora (Drucker, 2013) y no como productora, papel que sigue estando reservado al hombre y que se puede visualizar al presentarse la mujer, en los libros de texto, como la que va al supermercado, a la tienda de moda o la que lleva a los niños/as al colegio. Esto se aleja de lo que se debería plantear en la escuela sobre el papel de la mujer en la sociedad actual.

Para llevar a cabo esta investigación, se estudió la realidad de los centros educativos, teniendo en cuenta el contexto social actual y el papel de la educación para la igualdad en nuestra sociedad. Posteriormente, se realizó una propuesta de intervención enfocada en los docentes como agentes del cambio, desde una perspectiva multidisciplinar e integradora con la tecnología como cimiento de esta, que nos ha reportado la posibilidad de crear "Edito $x$ Ellas".

\section{Aproximación cultural}

La problemática actual que se plantea en la sociedad con el creciente número de casos de violencia de género es fruto de la evolución histórica y las transformaciones sociales, culturales, económicas y políticas de la actual sociedad posmoderna. Estos elementos son intrínsecos en nuestra comunidad desde tiempos inmemoriales y, con la aparición de las Tecnologías de la Información y Comunicación (en adelante, TIC) y las distintas 
transformaciones sociales, han devenido que aparezcan nuevas formas de violencia en distintos escenarios, pero que, esencialmente, parten de una misma lógica y raíz estructural: el patriarcado. Estos, como señala Salguero (2012, en Sánchez, 2012), tienen su origen en la educación, poniendo de manifiesto el papel relevante que, como docentes, debemos llevar a cabo en las aulas.

Si bien es cierto que, la Constitución Española de 1978, la Ley Orgánica 8/2013, para la mejora de la calidad educativa (en adelante, LOMCE) ${ }^{1}$ y la Ley Orgánica 3/2007, para la igualdad efectiva de mujeres y hombres, ${ }^{2}$ respecto de su aplicación y aporte al constructo identitario del alumnado desde la escuela primaria, ha evidenciado las grandes dificultades y limitaciones que se presentan a las mujeres, a tenor de los resultados reflejados en nuestra sociedad en la que, en pleno siglo XXI e inmersos en una civilización posmoderna, globalizada y tecnológica, el hombre sigue ocupando un papel de dominancia sobre la mujer. Esto supone, como afirma Casares (2008), una fuerte fragmentación social patente en todas las esferas visibles por nuestro alumnado.

En la actualidad, vivimos en un contexto histórico denominado posmodernidad en el que cabe destacar, tal como afirman Builes y Bedoya (2011), el papel cambiante de la familia en las sociedades posmodernas donde esta ha dejado de ser una institución pública, en la que la autoridad recaía en el padre o en los familiares varones, a ser una institución privada atravesada por la lógica patriarcal. Sin embargo, históricamente, la figura masculina de la familia y el poder que ejerce sobre el sexo femenino, continúa poniendo de manifiesto una distribución desigual de poder en las familias y que es extensible a la sociedad.

Daros (2014), siguiendo a Erazo y Maurel, afirma que, en los últimos 30 años en Occidente, la mujer ha conquistado el poder de ser dueña de ella misma, de su cuerpo, de su conocimiento y de su actividad. Lipovestsky (1999 en Daros, 2014) por el contrario, señala que este cambio no implica que se borre el pasado, y que se crea que se ha instaurado un

\footnotetext{
${ }^{1}$ Ley Orgánica 8/2013, de 9 de diciembre, para la mejora de la calidad educativa. Boletín Oficial del Estado, núm. 295, Madrid, España, 10 de diciembre de 2013.

Recuperado de: https://www.boe.es/boe/dias/2013/12/10/pdfs/BOE-A-2013-12886.pdf

${ }^{2}$ Ley Orgánica 3/2007, de 22 de marzo, para la igualdad efectiva de mujeres y hombres. Boletín Oficial del Estado, núm. 71, Madrid, España, 23 de marzo de 2007.

Recuperado de: https://www.boe.es/boe/dias/2007/03/23/pdfs/A12611-12645.pdf
} 
modelo de igualdad y equidad entre sexos y roles, sino que la mujer ya no es definida por la mirada del hombre y sus necesidades.

En definitiva, se puede afirmar que el papel de la mujer en la sociedad posmoderna no solo se ciñe al rol de ésta como ama de casa, sino que avanza hacia el derecho a la libertad, la toma de decisiones propias y la equidad entre ambos sexos. A pesar de ello, este derecho y su adquisición, así como las leyes que lo regulan, no implican una igualdad efectiva y real en la actual sociedad posmoderna, globalizada y ligada al mass media ${ }^{3}$.

\section{El centro educativo como reflejo de una sociedad patriarcal}

Lagarde (2012, en Sánchez, 2012) apunta que el patriarcado, es el poder que se fundamenta en la dominación genealógica de los hombres sobre sus mujeres y sus descendientes y que se caracteriza por una serie de costumbres, tradiciones, actitudes, normas y hábitos familiares y escolares cuya enseñanza y aprendizaje se transmite de manera implícita y generacional (Paredes, 2012, en Sánchez, 2012). En la escuela, de manera general, se transmitirán con actos, gestos y símbolos que no están recogidos en la legislación educativa y que se realizan de manera inconsciente y no premeditada mientras se imparten los contenidos del currículo oficial. Esto también se transmite a través de los elementos que se presentan en los libros de texto oficiales y que las editoriales plantean bajo el amparo del marco educativo legal de nuestro país.

Así mismo y, siguiendo a Paredes (2014), el patriarcado define los roles y los estereotipos de género, siendo la primera estructura de dominación y subordinación de la historia que ejerce una dominancia opresora sobre la mujer.

En este sentido, tal como señala Álamo (2012), el patriarcado es un fenómeno universal que ha existido a lo largo del tiempo en las diferentes culturas y está presente de múltiples formas en la escuela actual.

A pesar de que el marco legislativo español aboga por una educación en la igualdad y para la igualdad, poniendo, a priori, todas las herramientas necesarias para que deje de ser una

${ }^{3}$ Conjunto de medios de comunicación de masas (televisión, cine, radio y prensa). 
utopía y se convierta en una realidad efectiva, el reflejo que nos vierten nuestras aulas dista bastante del fenómeno socio estructural que debiera ser la igualdad entre hombres y mujeres.

Por ello, en la sociedad actual, la mujer sigue anclada en el modelo patriarcal de producción de mercancías, en las que las formas de opresión han tornado en dificultar el acceso a los puestos de mando y liderazgo, la realización de tareas para las que están sobrecualificadas, la innegable brecha salarial, así como, la concepción de la mujer como objeto comercial. Es por ello que se hace fundamental que el profesorado ejecute desde las aulas un rol facilitador para activar el cambio.

\section{El currículum oculto}

Si entendemos que el currículo es el marco normativo que regula y determina los elementos del proceso de enseñanza y aprendizaje para cada enseñanza y etapa educativa, podemos afirmar, siguiendo a Moya (2011), que el currículo oculto está formado por aquellos elementos que pueden formar parte del currículo, pero no de la enseñanza. Es decir, por aquellos elementos que no se recogen en el plan de estudio, sino que van implícitos en él, que se transmiten de manera inconsciente y están cargados de valores y actitudes subjetivas que juegan un papel fundamental en el desarrollo integral del alumno.

Acevedo (2010) define el currículo oculto como el conjunto de normas, costumbres, creencias, lenguajes y símbolos que se manifiestan en la estructura y el funcionamiento de la institución y la comunidad educativa. Como aquello que se ve y se oye. Aquello que se asimila de manera inconsciente al ser transmitido sin una intencionalidad firme.

Es aquello que Stenhouse (2007) asocia a la intención, a lo que no está públicamente reconocido y escapa del control de la escuela.

En definitiva, el currículo oculto se desarrolla de una manera inconsciente y que, pese a no estar escrito, en ocasiones supera la fortaleza del currículo formal por el modo y forma de ser transmitido y vivenciado por el alumnado, $y$, por tanto, juega un papel fundamental en la línea que se trabaja, las diferencias de género y el modo que son tratadas en la escuela. 


\section{Objetivo del estudio}

El objetivo fundamental de esta investigación fue describir aquellos elementos implícitos que se transmiten en el aula a través del currículo oculto y los libros de Educación Primaria. Con los datos recabados se quiso diseñar una aplicación para dispositivos móviles que permita reportar los elementos machistas que los docentes encuentren en los libros de texto.

\section{Método}

El desarrollo de este estudio se llevó a cabo en dos etapas. En primer lugar, se desarrolló una investigación mixta en la que se utilizó tanto la metodología cuantitativa como la cualitativa para obtener una mayor información acerca de acciones pedagógicas sexistas a través del currículum oculto y los libros de texto de $5^{\circ}$ y $6^{\circ}$ de Educación Primaria.

Por otro lado, para el diseño experimental de la aplicación se realizó un prototipo funcional (tipo maqueta o mockup), a modo de objeto simulado, a través de una página web de diseño libre.

\section{Desarrollo}

Para abordar el estudio desde un enfoque cuantitativo, se realizó una encuesta estructurada de elaboración propia con preguntas de respuesta dicotómica cerrada y respuestas de elección múltiple. La encuesta se diseñó teniendo en cuenta las siguientes variables: sexo, lenguaje sexista, estereotipos, educación segregada, trato igualitario, liderazgo y medidas educativas para la igualdad.

Esto nos permitió, desde una perspectiva cuantitativa, controlar las variables y medir los resultados de forma numérica y objetiva (Cook y Reichardt, 1986).

Por otro lado, para abordar la perspectiva cualitativa, se elaboró un estudio de casos sobre la realidad que concierne a los centros educativos. Siguiendo a Ceballos (2009), consistió en la observación, descripción, explicación y juicio de una situación real a través del visionado in situ del contexto educativo del aula y de los libros de texto usados en el centro ya que, según Flick (2014), esta metodología nos permitiría obtener unos resultados a partir de las actividades y expresiones de las personas en su contexto natural. 
En segundo lugar, se realizó un prototipo funcional (tipo maqueta o mockup) de la aplicación "Edito x Ellas", a modo de objeto simulado y como elemento experimental, a través de una página web de diseño libre, con una implementación sencilla, que permitiese verificar el concepto de la aplicación y la susceptibilidad de ser explotada de manera útil en el mercado comercial.

\section{Resultados}

Los resultados obtenidos apuntan, por un lado, que los docentes, de manera inconsciente, realizaban prácticas sexistas y que con su discurso continuaban contribuyendo a la desigualdad.

Algunos de los resultados obtenidos en esta investigación mostraron que casi un $60 \%$ de las mujeres objeto de estudio reconocieron utilizar siempre el género masculino como global, mientras que, casi el $70 \%$ de los hombres encuestados, expusieron usarlo a veces.

En cuanto a la firmeza con la que reprenden a los niños y niñas, en un $80 \%$, el profesorado respondió que sí reprenden del mismo modo a niños y niñas, frente al 20\% que manifestó que no lo hace.

En relación al currículo oculto, un 50\% de los encuestados respondió que sí lo tienen en cuenta, mientras que la otra mitad afirmó no tenerlo en cuenta en sus decisiones metodológicas y logísticas. De este modo, casi el $70 \%$ de los hombres encuestados afirmaron que no valoran el currículo oculto de los libros, al igual que al $42 \% 86 \%$ de las mujeres encuestadas.

Así mismo, al analizar los libros de texto de $5^{\circ}$ y $6^{\circ}$ de Primaria observamos que estos presentan contenidos machistas que asignan a la mujer un rol estereotipado, por lo que se alejan de lo estipulado por la legislación vigente en España. 


\subsection{Resultados cuantitativos}

En la encuesta realizada al profesorado encontramos que a la pregunta: “ ¿utiliza el plural masculino para englobar en él también a las niñas, por ejemplo, usa: los alumnos?" Un $40 \%$ ha respondido que siempre usan el plural masculino, otro $40 \%$ reconoce que lo usa a veces y, el $20 \%$ de los encuestados, afirman que nunca usa el género masculino como global (véase tabla 1 y figura 1 ).

Al cruzar los datos estadísticos con datos demográficos observamos que las mujeres encuestadas reconocieron, en casi un 60\%, usar siempre el género masculino como global frente a otras opciones, mientras que casi el $70 \%$ de los hombres encuestados reconocieron usarlo a veces.

Tabla 1. Uso general del plural masculino

HOMBRE MUJER TOTAL

$\begin{array}{llll}\text { A } & 0,00 \% & 57,14 \% & 40,00 \% \\ \text { B } & 66,67 \% & 28,57 \% & 40,00 \% \\ \text { C } & 33,33 \% & 14,29 \% & 20,00 \%\end{array}$

$\begin{array}{llll}\text { Total general } \quad \mathbf{1 0 0 , 0 0 \%} & \mathbf{1 0 0 , 0 0 \%} & \mathbf{1 0 0 , 0 0 \%}\end{array}$

Fuente: Elaboración propia

A la segunda cuestión sobre si realizaban comentarios del tipo: "esto parece un gallinero", “deja de llorar que pareces una niña”, los docentes respondieron, en un 90\%, que no hacen ese tipo de comentarios y, al cruzarlo con datos por sexos, observamos que el $100 \%$ de los hombres han respondido que no hacen ese tipo de comentarios, mientras que, al menos un $14 ’ 29 \%$ de las mujeres reconoció hacer esos comentarios (véase tabla 2 y figura 2). 
Tabla 2. Frecuencia con la que hacen comentarios sexistas

HOMBRE MUJER TOTAL
A
$0,00 \%$
$14,29 \%$
$10,00 \%$
B
$100,00 \%$
$85,71 \%$
$90,00 \%$

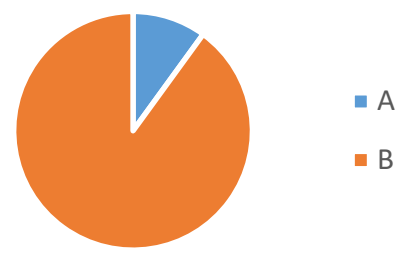

Figura 2. Frecuencia con la que hacen comentarios sexistas.

Fuente: Elaboración propia

$\begin{array}{llll}\text { Total general } \quad \mathbf{1 0 0 , 0 0 \%} & \mathbf{1 0 0 , 0 0 \%} & \mathbf{1 0 0 , 0 0 \%}\end{array}$

Fuente: Elaboración propia

En la pregunta 3, "en cuanto a la formulación de las preguntas en el aula, ¿tienen el mismo tema, complejidad e interés las que le realiza a los alumnos que las que realiza a las alumnas? Por ejemplo, ¿le pregunta a una alumna por un partido de fútbol del día anterior?" El 90 \% de los encuestados respondieron que sí realizaban el mismo tipo de preguntas a niños y niñas, mientras que un $10 \%$ admitió que no realizaba las mismas cuestiones a los distintos sexos (véase tabla 3 y figura 3 ).

En el análisis diferenciado por género, pudimos observar como el 100\% de los hombres encuestados manifestó realizar el mismo tipo de preguntas, mientras el 14’29\% de las mujeres admitió no realizar las mismas preguntas. 
MADAY DOMÍNGUEZ SÁNCHEZ; ARMINDA ÁlAMo Bolaños. Edito $x$ ellas: una app que conecta educación e igualdad de género

Tabla 3. Tipo de preguntas que realiza

\begin{tabular}{llll}
\hline & HOMBRE & MUJER & TOTAL \\
\hline A & $100,00 \%$ & $85,71 \%$ & $90,00 \%$ \\
& & & \\
B & $0,00 \%$ & $14,29 \%$ & $10,00 \%$
\end{tabular}

Figura 3. Tipo de pregunta que realiza.

Total general $\quad \mathbf{1 0 0 , 0 0 \%} \quad \mathbf{1 0 0 , 0 0 \%} \quad \mathbf{1 0 0 , 0 0 \%} \quad$ Fuente: Elaboración propia

Fuente: Elaboración propia

En la pregunta 4 sobre si reprende con igual firmeza a los niños y niñas, en un $80 \%$, el profesorado respondió que sí reprendían del mismo modo a niños y niñas frente al $20 \%$ que manifestó que no lo hace.

De este modo, todos los profesores afirmaron que reprendían por igual mientras que, el $28{ }^{\prime} 57 \%$ de las profesoras afirmaron que no reprenden con la misma firmeza a los alumnos que a las alumnas (véase tabla 4 y figura 4 ).

Tabla 4. Forma en la que reprende al alumnado

\begin{tabular}{llll}
\hline & HOMBRE & MUJER & TOTAL \\
\hline A & $100,00 \%$ & $71,43 \%$ & $80,00 \%$ \\
B & $0,00 \%$ & $28,57 \%$ & $20,00 \%$ \\
Total general & $\mathbf{1 0 0 , 0 0 \%}$ & $\mathbf{1 0 0 , 0 0 \%}$ & $\mathbf{1 0 0 , 0 0 \%}$ \\
\hline
\end{tabular}

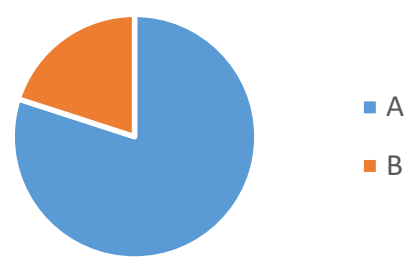

Figura 4. Forma en la que reprende al alumnado.

Fuente: Elaboración propia

Fuente: Elaboración propia 
Al ser preguntados por "cuando los alumnos y alumnas deben trabajar en equipo, ¿a quién nombra como líder?" Todo el profesorado (véase tabla 5 y figura 5) respondió que nombraban, de manera indiferente, a unos u otras como líderes de equipo cuando estos tienen que trabajar engrupo.

Tabla 5. ¿A quién nombra como líder?

\begin{tabular}{llll}
\hline & HOMBRE & MUJER & TOTAL \\
\hline $\mathbf{A}$ & $0,00 \%$ & $0,00 \%$ & $0,00 \%$ \\
$\mathbf{B}$ & $0,00 \%$ & $0,00 \%$ & $0,00 \%$ \\
$\mathbf{C}$ & $100 \%$ & $100 \%$ & $100 \%$ \\
Total general & $\mathbf{1 0 0 , 0 0 \%}$ & $\mathbf{1 0 0 , 0 0 \%}$ & $\mathbf{1 0 0 , 0 0 \%}$ \\
\hline
\end{tabular}

Figura 5. ¿A quién nombra como líder? Fuente: Elaboración propia

Fuente: Elaboración propia

Ante la pregunta de si "cuando se reúnen con el equipo docente para tomar decisiones metodológicas y logísticas, ¿tienen en cuenta el currículo oculto de los libros?” En un 50\%, los encuestados respondieron que sí tenían en cuenta el currículo oculto, mientras que la otra mitad afirmó no tenerlo en cuenta en sus decisiones metodológicas y logísticas. De este modo, casi el 70\% de los hombres encuestados afirmaron que no valoran el currículo oculto de los libros, al igual que al 42 ’6\% de las mujeres preguntadas (véase tabla 6 y figura 6 ). 
Tabla 6. ¿Tiene en cuenta el currículo oculto?

\begin{tabular}{|c|c|c|c|c|}
\hline & HOMBRE & MUJER & TOTAL & $=\mathrm{A}$ \\
\hline $\mathbf{A}$ & $33,33 \%$ & $57,14 \%$ & $50,00 \%$ & \\
\hline B & $66,67 \%$ & $42,86 \%$ & $50,00 \%$ & Figura 6. ¿Tiene en cuenta el currículo oculto? \\
\hline $\begin{array}{l}\text { Total } \\
\text { general }\end{array}$ & $100,00 \%$ & $100,00 \%$ & $100,00 \%$ & Fuente: Elaboración propia \\
\hline
\end{tabular}

Fuente: Elaboración propia

En la pregunta 7, al ser preguntados si “ ¿estaría dispuesto a colaborar para mejorar las líneas editoriales de los libros de texto en lo que a igualdad se refiere?" Un $70 \%$ de los docentes afirmó que sí participarían porque es un tema en el que todos nos debemos implicar. El 20\% afirma que no colaboraría pues para eso ya están las editoriales y, un10\% de los encuestados, afirmó que los libros no necesitan modificaciones porque están hechos bajo el amparo de la ley actual (véase tabla 7 y figura7).

Al cruzar los resultados con los datos demográficos, observamos que el $100 \%$ de los hombres se prestarían a colaborar con las editoriales para mejorar los contenidos frente al $57^{\prime} 14 \%$ de las mujeres. El 28'57\% de estas afirmaron que no colaborarían, pues es trabajo de las editoriales y, el 14'29\% de estas asegura que los libros no necesitan modificaciones. 
MAdAy Domínguez SÁnchez; Arminda Álamo Bolaños. Edito x ellas: una app que conecta educación e igualdad de género

Tabla 7. ¿Colaboraría para mejorar los libros?

\begin{tabular}{llll}
\hline & HOMBRE & MUJER & TOTAL \\
\hline A & $100,00 \%$ & $57,14 \%$ & $70,00 \%$ \\
$\mathbf{B}$ & $0,00 \%$ & $28,57 \%$ & $20,00 \%$ \\
$\mathbf{C}$ & $0,00 \%$ & $14,29 \%$ & $10,00 \%$ \\
Total & $\mathbf{1 0 0 , 0 0 \%}$ & $\mathbf{1 0 0 , 0 0 \%}$ & $\mathbf{1 0 0 , 0 0 \%}$ \\
general & & & \\
\hline
\end{tabular}

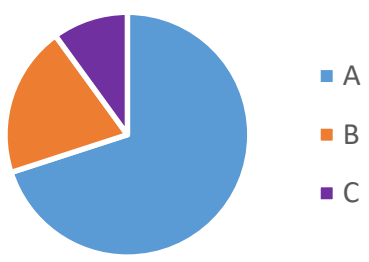

Fuente: Elaboración propia

Figura 7. ¿Colaboraría para mejorar los libros?

Fuente: Elaboración propia 
MADAy Domínguez SÁNCHEZ; ARMinda Álamo Bolaños. Edito x ellas: una app que conecta educación e igualdad de género

\section{2. Resultados cualitativos}

Tabla 8. Observaciones sobre los libros de Lengua y Matemáticas

\begin{tabular}{|c|c|c|c|c|}
\hline LIBRO & Lengua $5^{\circ}$ & Lengua $6^{\circ}$ & Matemáticas $5^{a}$ & Matemáticas $6^{\circ}$ \\
\hline Portada & Aparece un niño & Aparece una niña & $\begin{array}{c}\text { Símbolos } \\
\text { matemáticos }\end{array}$ & $\begin{array}{c}\text { Símbolos } \\
\text { matemáticos }\end{array}$ \\
\hline $\begin{array}{l}\text { Quiénes aparecen más } \\
\text { enlas }\end{array}$ & $\begin{array}{l}\text { Más niños que } \\
\text { niñas }\end{array}$ & $\begin{array}{c}\text { Más niños que } \\
\text { niñas }\end{array}$ & $\begin{array}{l}\text { Mismo número de } \\
\text { niños y niñas }\end{array}$ & $\begin{array}{l}\text { Mismo número de } \\
\text { niños y niñas }\end{array}$ \\
\hline $\begin{array}{l}\text { ilustraciones } \\
\text { infografías }\end{array}$ & & & & \\
\hline $\begin{array}{l}\text { Uso del masculino } \\
\text { como genérico }\end{array}$ & Sí. Siempre. & Sí. Siempre. & Sí. Siempre. & Sí. Siempre. \\
\hline $\begin{array}{l}\text { Autores } \\
\text { personajes } \\
\text { relevantes }\end{array}$ & $\begin{array}{l}\text { Más autores que } \\
\text { autoras en las } \\
\text { lecturas iniciales }\end{array}$ & $\begin{array}{l}\text { Más autores que } \\
\text { autoras en las } \\
\text { lecturas iniciales }\end{array}$ & - & \\
\hline $\begin{array}{l}\text { Tipo de } \\
\text { actividad/acción que } \\
\text { realiza el que aparece } \\
\text { en las }\end{array}$ & $\begin{array}{l}\text { La mujer aparece } \\
\text { como princesa, } \\
\text { bruja o ama de } \\
\text { casa. }\end{array}$ & & $\begin{array}{l}\text { Niños y niñas } \\
\text { realizan las } \\
\text { mismas } \\
\text { actividades/ }\end{array}$ & $\begin{array}{lr}\text { Niños y niñas } \\
\text { realizan } \\
\text { mismas } \\
\text { actividades/ }\end{array}$ \\
\hline ilustraciones & & & acciones & acciones \\
\hline
\end{tabular}

Fuente: Elaboración propia 
MAdAy Domínguez SÁNChez; Arminda Álamo Bolaños. Edito x ellas: una app que conecta educación e igualdad de género

Tabla 9. Observaciones sobre los libros de Ciencias Sociales y Ciencias Naturales

\begin{tabular}{|c|c|c|c|c|}
\hline LIBRO & $\begin{array}{c}\text { Ciencias Sociales } \\
\mathbf{5}^{\mathbf{0}}\end{array}$ & $\begin{array}{c}\text { Ciencias Sociales } \\
6^{\circ}\end{array}$ & $\begin{array}{c}\text { Ciencias } \\
\text { Naturales } 5^{\circ}\end{array}$ & $\begin{array}{c}\text { Ciencias } \\
\text { Naturales }^{\circ}\end{array}$ \\
\hline Portada & Motivos sociales & Motivos sociales & Motivos naturales & Motivos naturales \\
\hline $\begin{array}{l}\text { Quiénes aparecen } \\
\text { más en las } \\
\text { lustraciones }\end{array}$ & $\begin{array}{l}\text { Más hombres que } \\
\text { mujeres. }\end{array}$ & $\begin{array}{l}\text { Más hombres que } \\
\text { mujeres. }\end{array}$ & $\begin{array}{l}\text { Más niños que } \\
\text { niñas }\end{array}$ & $\begin{array}{l}\text { Más niños que } \\
\text { niñas }\end{array}$ \\
\hline $\begin{array}{l}\text { Uso del masculino } \\
\text { como genérico }\end{array}$ & Sí. Siempre. & Sí. Siempre. & Sí. Siempre. & Sí. Siempre. \\
\hline $\begin{array}{l}\text { Autores } \\
\text { personajes } \\
\text { relevantes }\end{array}$ & $\begin{array}{l}\text { Más personajes } \\
\text { históricos } \\
\text { hombres. }\end{array}$ & $\begin{array}{l}\text { Más personajes } \\
\text { hombres. }\end{array}$ & $\begin{array}{l}\text { Todos los } \\
\text { científicos } \\
\text { relevantes son }\end{array}$ & $\begin{array}{l}\text { Todos los } \\
\text { científicos } \\
\text { relevantes son }\end{array}$ \\
\hline & & & hombres & $\begin{array}{l}\text { hombres. Solo una } \\
\text { ilustración } \\
\text { mujer en el } \\
\text { laboratorio. }\end{array}$ \\
\hline
\end{tabular}

\begin{tabular}{|c|c|c|c|c|}
\hline $\begin{array}{l}\text { d/acción } \\
\text { liza el que }\end{array}$ & $\begin{array}{l}\text { Los hombres son } \\
\text { pintores, artistas o } \\
\text { personajes } \\
\text { importantes para la } \\
\quad \text { historia. La } \\
\text { mujer, } \\
\text { prácticamente, no } \\
\text { aparece } \\
\text { representada en el } \\
\text { bloque de Historia. }\end{array}$ & $\begin{array}{l}\text { Todos los hitos } \\
\text { importantes de } \\
\text { innovación técnica } \\
\text { son atribuidos a } \\
\text { hombres en el libro. } \\
\text { La mujer, } \\
\text { prácticamente, no } \\
\text { aparece } \\
\text { representada. }\end{array}$ & $\begin{array}{lr}\text { Los } & \text { hombres } \\
\text { aparecen } & \text { como } \\
\text { obreros, operarios } \\
\text { o jefes. Son los que } \\
\text { manejan r la } \\
\text { maquinaria y los } \\
\text { equipos. }\end{array}$ & $\begin{array}{l}\text { La mujer aparece } \\
\text { embaraza en los } \\
\text { contenidos de la } \\
\text { reproducción. }\end{array}$ \\
\hline
\end{tabular}

Fuente: Elaboración propia 
En las tablas 8 y 9, que muestran los resultados cualitativos del análisis de los libros de texto de $5^{\circ}$ y $6^{\circ}$ de Primaria, se observa que, pese a mostrar portadas en las que aparecen motivos relacionados con el contenido de las asignaturas o niños y niñas indistintamente, en su interior, se detecta contenido sexista.

Se observa la presencia de un mayor número de niños que de niñas en las infografías y los hitos históricos relatados son llevados a cabo por hombres. Así mismo, podemos observar que tanto los autores de los textos como del propio libro son, en su mayoría, hombres. Se usa, de manera generalizada el masculino en todos los textos y queda patente como la mujer queda relegada a ser muestra de contenidos referidos a tareas del hogar, la gestación o los mundos imaginarios de brujas y princesas.

\section{9. "Edito x Ellas"}

Siguiendo Rebollo (2013), para accionar el cambio educativo en materia de igualdad es necesario que el docente "desarrolle buenas prácticas" $(2013,5)$, que pongan en marcha acciones, hábitos y prácticas igualitarias que modifiquen la conducta automatizada intrínseca que, de manera inconsciente, se transmite con la docencia. Así mismo, es fundamental poner de manifiesto la necesidad de transformar, no solo lo que transmitimos de manera verbal e inconsciente, sino lo que viene implícito en los libros de texto con los que se trabaja en Educación Primaria.

Para poner en marcha este cambio, se ha diseñado una aplicación para dispositivos móviles con sistema Android, "Edito x Ellas", que permite a los docentes, de un modo intuitivo y sencillo de pantallas, enviar reportes a las editoriales al detectar en los libros estas acciones sexistas que favorezcan desigualdades de género como las descritas en nuestro estudio. 


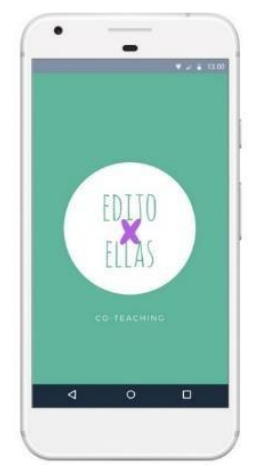

Para ello, el docente deberá seguir los pasos de la interfaz de usuario en la que tendrá que:

- Validar los datos requeridos (e-mail, Comunidad Autónoma y editorial).

- Escanear el código ISBN del libro de modo que obtenga una fotografía de este.

- Fotografiar la página en la que encuentran el contenido.

- $\quad$ Enviar el reporte a la editorial.

Para el diseño experimental propuesto para la aplicación, se ha realizado un prototipo funcional, a modo de objeto simulado, a través de una página web de diseño libre con una implementación sencilla que permitiese verificar el concepto de la aplicación y la susceptibilidad de ser explotada de manera útil. Esta se ha podido instalar en diferentes dispositivos para comprobar su usabilidad y las posibles mejoras que se pueden implementar para conseguir un funcionamiento óptimo que contribuya con el objeto de su creación.

Además, para el correcto funcionamiento de esta aplicación, se necesita un acuerdo previo con las editoriales en el que, además de la colaboración docente-centro-editorial, se establezca un compromiso riguroso de mejora del contenido de los libros por parte de estas últimas en favor del cumplimiento real y firme de la legislación vigente en materia de igualdad. 


\subsection{Diseño de la interfaz de la app "Edito x Ellas"}

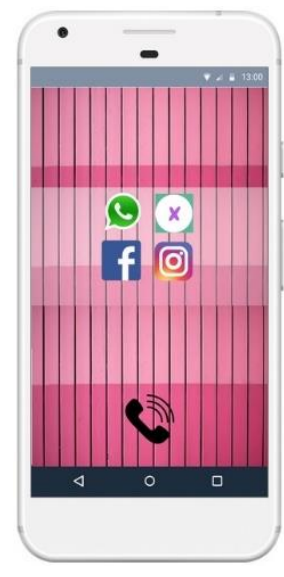

1. El usuario debe situarse en la pantalla principal de su dispositivo móvil y seleccionar el icono de nuestra aplicación.

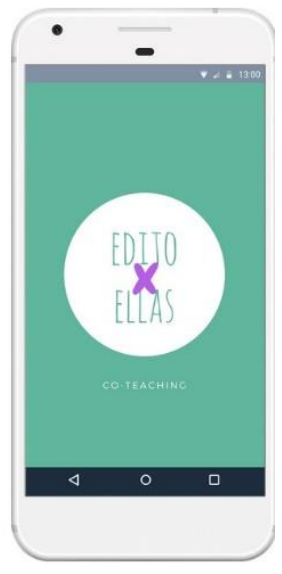

2. Al abrir la aplicación les aparecerá la siguiente imagen

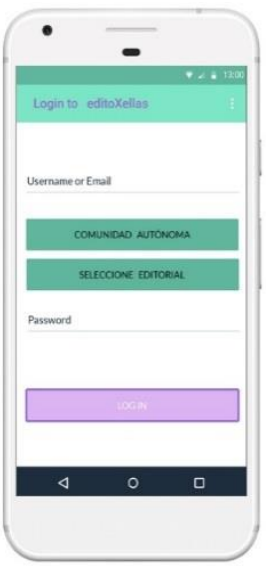

3. Al tocar la pantalla predictiva de la imagen anterior, aparecerá un formulario que el usuario debe rellenar con: nombre de usuario, Comunidad Autónoma (a través de un desplegable) y Editorial del libro de texto

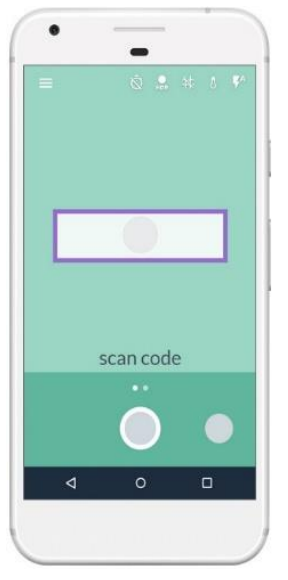

4. Una vez terminado el formulario, salta una pantalla para escanear el código del libro tomando una foto 


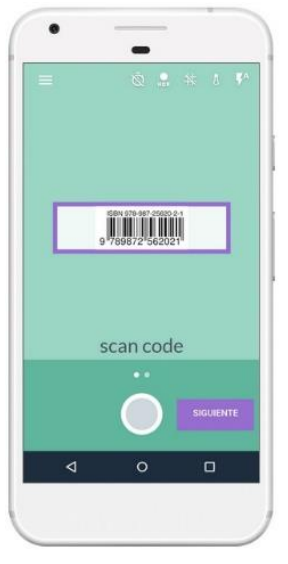

5. Con el código escaneado, se activa la pestaña siguiente.

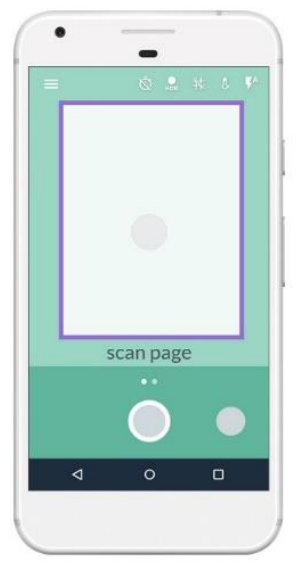

6. Tras la pantalla anterior, salta otra para escanear, en esta ocasión, la página del libro en la que aparece el contenido sexista del mismo modo que hicimos con el código del libro, tomando una foto.
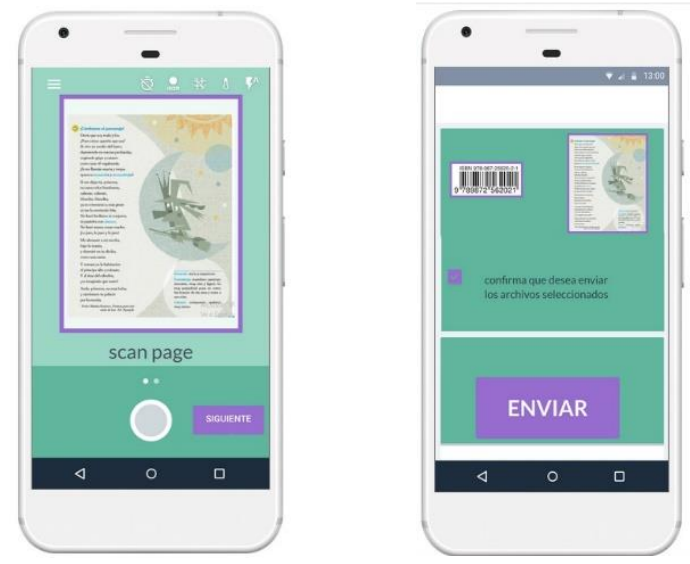

7. Con la página del libro escaneada, se activa la pestaña siguiente.
8. Una vez comprobado que el código y la página están correctos, marcamos la pestaña confirmar y enviar para que el reporte llegue a la editorial.

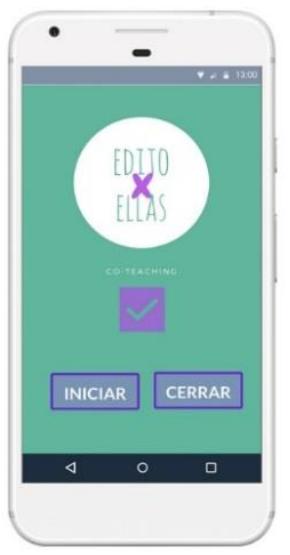

9. Una vez completado el envío, salta una pantalla de confirmación que ofrece volver al inicio para enviar un nuevo reporte o salir de la aplicación. 


\section{Discusión}

Los resultados de nuestro estudio mostraron datos contradictorios entre lo que se dice y lo que se hace en las aulas de Educación Primaria.

Al analizar la pregunta del uso del género masculino como genérico, nos dimos cuenta de que el $80 \%$ del profesorado lo utilizan a menudo o siempre en sus prácticas docentes y que son más los hombres que generalizan sobre el término.

Por otro lado, al preguntar si realizaban comentarios discriminatorios como los descritos en la pregunta 2 de nuestra encuesta del tipo "esto parece un gallinero" o "deja de llorar que pareces una niña" sorprendió que el 100\% de los hombres rechace haber hecho uso de estos comentarios mientras que un 14 ’29\% de las profesoras reconocían esta práctica, pues si cruzamos los datos cuantitativos con las observaciones realizadas en el centro, se nos mostraba que los profesores no han dicho del todo la verdad, pues son frases más habituales de lo que reconocen en la encuesta.

En la línea de la pregunta anterior, tan solo un 28'57\% de las profesoras afirmaron que no reprenden igual a los niños que a las niñas, mientras que el 100\% de los maestros aseguraron mostrar la misma firmeza. Este dato se ha podido constatar en el estudio de casos llevado a cabo en el que las profesoras tratan con mayor dureza al alumnado varón y que, siguiendo a Cárdenas, Lay, González Calderón y Alegría (2010), hace incisión en "la figura de mujer como 'sexo débil' y necesitada de protección y cuidado" $(2010,130)$.

En lo referente a la cuestión sobre la revisión del currículo oculto, los resultados del cuestionario revelaron la verdadera realidad del centro. La mitad de los docentes afirmaron que no tienen en cuenta el currículo oculto a la hora de tomar decisiones metodológicas y logísticas cuando eligen los libros con los que trabajar, haciendo que, en el currículo oficial y el currículo oculto, se transmita el carácter subordinado de la dominación operante masculina, contribuyendo así a un aprendizaje que en palabras de Subirats (2016), "implica que los títulos académicos o los saberes adquiridos no tengan el mismo valor según los ostente un hombre o una mujer" $(2016,29)$. 
Esto supone una supeditación que la sociedad acepta como hecho normalizado y que repercute en el valor figurado de la mujer en el ámbito laboral.

Es decir, si el profesorado no revisa el material y continúa adoptando una posición secundaria en la lucha por la igualdad real, conseguirá, a través de las enseñanzas con libros de texto no revisados, que la desigualdad de género y la discriminación se pongan de manifiesto, favoreciendo a que la educación continúe sin cumplir, de manera rigurosa con lo descrito en la legislación y, más importante aún, con el requerimiento que la sociedad precisa.

Así mismo, sorprende el dato vertido en los resultados de la pregunta 7 donde el $70 \%$ del profesorado encuestado están dispuestos a colaborar con las líneas editoriales para favorecer la igualdad, pues resulta contradictorio que, pese a no revisar el currículo oculto de los libros, quieran colaborar para mejorarlo.

En las observaciones realizadas en los centros, se puso de manifiesto cómo estos arrastran una ideología tradicional en la que se hacen diferencias de género. El primer factor que llama la atención es la necesidad de algunos centros de diferenciar al alumnado con sus uniformes. En el Reglamento Interno de los centros se recoge explícitamente que el uso de falda para las alumnas y pantalón para los alumnos será obligatorio y considerado como una falta leve su incumplimiento.

Así mismo, una de las profesoras que pudimos observar manifestó, abiertamente, durante una clase de Ciencias Sociales que el hombre es más fuerte y dominante que la mujer y que este debe mantenerla mientras ella se ocupa de los niños y el hogar o realiza trabajos menores que le permitan el cuidado de la descendencia. Otros docentes, realizaban comentarios como "los niños no deben llorar" o "las niñas no deben jugar al fútbol”. Las alumnas son instadas a "comportarse como señoritas" poniendo de manifiesto la existencia de un profesorado, conservador en sus ideales, que eleva el poder de los niños para realizar ciertos trabajos y proyecta una imagen sobre el papel de la mujer que la relega a un estrato social inferior al del hombre. 
A los datos descritos con anterioridad, debemos añadir que los libros de texto, lejos de promover una educación en igualdad y para la igualdad, arrastran todavía y, pese al esfuerzo legislativo que se ha realizado en nuestro país, una identidad patriarcal que sitúa a la mujer como un objeto de validez reproductiva y no como ser social cooperante en la historia y la sociedad. Por tanto, estas diferencias deben ser tratadas desde dentro pues, tal como señala Bauman y Mazzeo (2012), una de las crisis en la educación está causada por las diferencias que existen en la sociedad siendo esta la que justifica la existencia de desigualdades derivadas de la movilidad social en la que estamos inmersos y que atañe a la educación en primera instancia. De este modo, tal como afirma Cobo (2011) "la educación en estos momentos históricos es una institución clave para el rearme ideológico y material del Patriarcado" $(2011,64)$. Es decir, la educación es clave para luchar contra la desigualdad de género $\mathrm{y}$, por ello, la labor de los docentes juega un papel fundamental en la reestructuración de la institución.

Por tanto, los docentes, las instituciones educativas y la sociedad debemos apostar por una educación por la igualdad y para la igualdad que se diferencie de las líneas de actuación que se han venido desarrollando en el tiempo y que, a tenor de los resultados vertidos en nuestro estudio, no han conseguido superar los cánones impuestos por la sociedad para los distintos roles de género. Para llevar a cabo este proceso en las instituciones educativas se hace necesario contar, no solo con la implicación docente, sino con la ayuda y colaboración de las editoriales educativas.

A partir del estudio realizado y los resultados obtenidos, se desprende el diseño de una intervención que pretende acciones educativas en el ámbito de la igualdad.

\section{Conclusiones}

Los principales problemas a los que la escuela tiene que hacer frente en materia de igualdad están relacionados, de manera directa, con lo que hemos definido en esta investigación como currículo oculto. A través de este, se perpetúa la transmisión de valores que sitúan a la mujer en un lugar de inferioridad social con respecto al hombre, encumbrando a este en su papel de dominio. En la escuela, el currículo oculto, se hace patente a través del discurso 
del profesorado y sus acciones, pero también, como ya hemos mencionado con anterioridad, a través del material didáctico con el que trabajamos.

Por tanto, hemos pretendido situar el foco de nuestro estudio en el profesorado pues es el agente que tiene la capacidad de accionar el cambio sobre la realidad que le rodea. Para ello se ha creado una intervención donde las TIC tienen un papel fundamental en el proceso pues, aunque la aparición de estas no ha favorecido que el papel de la mujer se refuerce y se aleje de la imagen simbólica que la historia le ha otorgado, resulta fundamental que, estando en la denominada Sociedad del Conocimiento, se luche contra las desigualdades haciendo uso de las herramientas que la TIC nos brinda para ello.

Se confirma que muchos libros de texto, lejos de promover una educación para la igualdad, arrastran todavía y, pese al esfuerzo legislativo que se ha realizado en nuestro país, un contenido machista que sitúa a la mujer como un objeto de validez reproductiva y no como ser social cooperante en la sociedad.

Por tanto, estas diferencias deben ser tratadas desde dentro pues, como apuntan algunos autores, una de las crisis en la educación está causada por las diferencias que existen en la sociedad siendo esta la que justifica la existencia de desigualdades derivadas de la movilidad social y que atañe a la educación en primera instancia.

De este modo, podemos afirmar que la educación es en la actualidad un instrumento clave para modificar la ideología tradicional y material del Patriarcado. Es decir, la educación es clave para luchar contra la desigualdad de género y, por ello, los docentes juegan un papel fundamental en la reestructuración de la institución.

Para llevar a cabo este proceso en las instituciones educativas, se hace necesario contar con la ayuda y colaboración de las editoriales educativas, a las que debemos pedir que se sumen a la lucha por la igualdad.

Se precisa una estrecha colaboración entre la triada escuela-editoriales-sociedad, sin olvidar que es crucial el papel del profesorado en la detección de los elementos sexistas intrínsecos en los libros de texto como currículo oculto, pues son ellos los agentes capaces de accionar 
el cambio en la creciente e imparable Sociedad del Conocimiento, haciendo uso de las TIC y usando "Edito x Ellas" en favor de una Educación por y para la Igualdad.

\section{Referencias bibliográficas}

Acevedo, Eugenia. (2010). "El currículo oculto en las enseñanzas formales. Aspectos menos visibles a tener en cuenta para una educación no sexista”, Revista digital de profesionales de la enseñanza, 11.

Recuperado de: https://www.feandalucia.ccoo.es/docu/p5sd7590.pdf

Álamo Bolaños, Arminda. (2015). "Las diferencias de género, la diversidad cultural, religiosa y valores morales en la lógica de la sociedad de la postmodernidad", en: Álamo, A. y Almeida, A., Manuales Universitarios de Teleformación Grado en Educación Primaria n. ${ }^{\circ}$ 41: Educación para la Ciudadanía, Las Palmas de Gran Canaria, España: ULPGC, 125-158.

Bauman, Zygmunt \& Mazzeo, Rafe. (2012). Sobre educación en un mundo líquido: conversaciones con Ricardo Mazzeo. Barcelona: Paidós Ibérica.

Bello Crespo, Carmen; Bernal Regalado, Tomás; Lluva Mera, Carlos; Magarzo Jiménez, José Luis; J. \& Zaragoza García, Pilar. (2015). Lengua 5. Madrid: Anaya.

Builes Correa, María Victoria \& Bedoya Hernández, Mauricio. (2008). “La familia contemporánea: relatos de resiliencia y salud mental”, Revista Colombiana de Psiquiatría, vol. 37, 3, 344-353.

Recuperado de: https://www.redalyc.org/pdf/806/80611205005.pdf

Cárdenas, Manuel; Lay, Siu-Lyn; González, Carmen; Calderón Carlos \& Alegría, Isabel. (2010). "Inventario de sexismo ambivalente: adaptación, validación y relación con variables psicosociales", Revista Salud y Sociedad, vol. 1, 2, 125-135. Recuperado de : http://revistas.ucn.cl/index.php/saludysociedad/article/view/751/633 
Casares García, Esther. (2008). “La función de la mujer en la familia. Principales enfoques teóricos", Aposta: Revista de Ciencias Sociales, 36, enero-marzo, 2008, 1-20.

Recuperado de : http://www.apostadigital.com/revistav3/hemeroteca/ecasares.pdf

Ceballos Herrera, Froilán Antonio. (2009). "El informe de investigación con estudio de casos", Revista Internacional de Investigación en Educación, 2, 413-423. Recuperado de: http://www.redalyc.org/html/2810/281021548015/

Cobo Bedía, Rosa. (2011). “Educación para la libertad? Las mujeres ante la reacción patriarcal", Revista Interuniversitaria de Formación del Profesorado, 71, 63-72. Recuperado de: https://dialnet.unirioja.es/servlet/articulo?codigo=4110563

Cook, Tomas. \& Reichardt, Charles. (1986). Métodos cualitativos y cuantitativos en investigación evaluativa. Madrid: Ediciones Morata.

Daros, William. (2014). “La mujer posmoderna y el machismo”. Franciscanum 162, Vol. LVI, 107-129.

Recuperado de: $\quad$ http://www.scielo.org.co/scielo.php?pid=S0120$14682014000200005 \&$ script $=$ sci_arttext\&tlng=pt

Drucker, Peter. (2013). La sociedad poscapitalista. Buenos Aires: Editorial Sudamericana.

Flick, Uwe. (2014). El diseño de la investigación cualitativa. Madrid: Ediciones Morata.

Moya, José. (2011). Los procesos de enseñanza y aprendizaje: fundamentos disciplinares, en: Moya, J. y Rodríguez, J., Manuales Docente Universitario Grado en Educación Primaria $n^{\circ}$ 2: Organización, Procesos Educativos e Innovación, Las Palmas de Gran Canaria, España: ULPGC, 209-288.

Paredes, David. (2014). "Patriarcado y Feminismo. Relaciones de Poder y Perspectiva de Género. Desestructurando los mandatos de género de la masculinidad. Hombres en tránsito o proceso de cambio hacia la generación de masculinidades alternativas no violentas", Revista del Centro de Estudios Históricos Interdisciplinarios sobre las Mujeres (CEHIM), 
año 10, 10, nueva época, 179-193. Recuperado de : http://filo.unt.edu.ar/wpcontent/uploads/2015/11/TEMAS_MUJERES_010_12PAREDES HRRERA.pdf

Rebollo, María. (2013). "La innovación educativa con perspectiva de género. Retos y desafíos para el profesorado", Profesorado: Revista de currículum y formación del profesorado, vol. $\quad 17, \quad 3, \quad 3-8 . \quad$ Recuperado de: https://idus.us.es/xmlui/bitstream/handle/11441/43551/INNOVACI\%C3\%93N\%20EDUC ATIVA\%20CON\%20PERSPECTIVA\%20DE\%20G\%C3\%89NERO.pdf?sequence=1

Sánchez, Carmen. (2012). Mujeres en diálogo: Avanzando hacia la despatriarcalización en Bolivia, La Paz, Bolivia: Editora Presencia SRL. Recuperado de: http://www.ciudadaniatransformadora.org/imagen/noticias/56/56_pdflink1.pdf\#page=196

Stenhouse, Lawrence. (2007). Investigación y desarrollo del currículo (4ª edición). Madrid: Ediciones Morata.

Subirats, Marina. (2016). "De los dispositivos selectivos en la educación: el caso del sexismo", Revista de la Asociación de Sociología de la Educación, vol. 9, 1, 22-36. Recuperado de: https://ojs.uv.es/index.php/RASE/article/view/8401/7994 